\title{
Heuristic Algorithms for Solving the Generalized Vehicle Routing Problem
}

\author{
P.C. Pop, C. Pop Sitar, I. Zelina, V. Lupşe, C. Chira
}

\author{
Petrică Claudiu Pop, Ioana Zelina, Vasile Lupşe \\ North University of Baia Mare, Department of Mathematics and Informatics \\ Romania, V. Babeş, 430083, Baia Mare \\ E-mail: petrica.pop@ubm.ro, ioanazelina@yahoo.com,vasilelupse@yahoo.co.uk \\ Corina Pop Sitar \\ North University of Baia Mare, Department of Economics \\ Romania, V. Babeş, 430083, Baia Mare \\ E-mail: sitarcorina@yahoo.com \\ Camelia Chira \\ "Babes-Bolyai" University of Cluj-Napoca \\ Romania, M. Kogalniceanu, 400084, Cluj-Napoca \\ E-mail: cchira@cs.ubbcluj.ro
}

\begin{abstract}
The vehicle routing problem (VRP) is one of the most famous combinatorial optimization problems and has been intensively studied due to the many practical applications in the field of distribution, collection, logistics, etc.

We study a generalization of the VRP called the generalized vehicle routing problem (GVRP) where given a partition of the nodes of the graph into node sets we want to find the optimal routes from the given depot to the number of predefined clusters which include exactly one node from each cluster. The purpose of this paper is to present heuristic algorithms to solve this problem approximately. We present constructive algorithms and local search algorithms for solving the generalized vehicle routing problem.

Keywords: network design, combinatorial optimization, generalized vehicle routing problem, heuristic algorithms.
\end{abstract}

\section{Introduction}

Combinatorial optimization is a lively field of applied mathematics, combining techniques from combinatorics, linear programming, and the theory of algorithms, to solve optimization problems over discrete structures. The study of combinatorial optimization owes its existence to the advent of modern digital computer. Most currently accepted methods of solution to combinatorial optimization problems would hardly have been taking seriously 30 years ago, for the simple reason that no one could have carried out the computations involved. Moreover, the existence of digital computers has also created a multitude of technical problems of a combinatorial character.

Combinatorial optimization problems can be generalized in a natural way by considering a related problem relative to a given partition of the nodes of the graph into node sets, while the feasibility constraints are expressed in terms of the clusters. In this way, it is introduced the class of generalized combinatorial optimization problems. In the literature one finds generalized problems such as the generalized minimum spanning tree problem [15], the generalized traveling salesman problem, the generalized vehicle routing problem, the generalized (subset) assignment problem, etc. These generalized problems belong to the class of NP-complete problems, 
are harder than the classical ones and nowadays are intensively studied due to the interesting properties and applications in the real world, even though many practitioners are reluctant to use them for practical modeling problems because of the complexity of finding optimal or nearoptimal solutions.

The Generalized Vehicle Routing Problem (GVRP) is an extension of the Vehicle Routing Problem (VRP) and was introduced by Ghiani and Improta [4]. The GVRP is the problem of designing optimal delivery or collection routes, subject to capacity restrictions, from a given depot to a number of predefined, mutually exclusive and exhaustive node-sets (clusters). The GVRP can be viewed as a particular type of location-routing problem (see, e.g. Laporte [7], Nagy and Salhi [10]) for which several algorithms, mostly heuristics, exist.

Ghiani and Improta [4] showed that the problem can be transformed into a capacitated arc routing problem (CARP) and Baldacci et al. [1] proved that the reverse transformation is valid. Recently, Pop [14] provided a new efficient transformation of the GVRP into the classical vehicle routing problem (VRP). In 2003, Kara and Bektas [5] proposed an integer programming formulation for GVRP with a polynomially increasing number of binary variables and constraints and in 2008 Kara and Pop [6] presented two integer linear programming formulations for GVRP with $O\left(n^{2}\right)$ binary variables and $O\left(n^{2}\right)$ constraints, where $n$ is the number of customers which are partitioned into a given number of clusters. As far as we know, the only specific algorithm for solving the GVRP was developed by Pop et al. [13] and was based on ant colony optimization.

The complexity of obtaining optimum or even near-optimal solutions for the generalized combinatorial optimization problems may lead to the development of:

- efficient transformations of the generalized combinatorial optimization problems into classical combinatorial optimization problems $[4,13]$;

- heuristic and metaheuristic algorithms [11].

The aim of this paper is to describe three classes of heuristic algorithms for solving approximately the generalized vehicle routing problem.

\section{Definition of the GVRP}

Let $G=(V, A)$ be a directed graph with $V=\{0,1,2, \ldots, n\}$ as the set of vertices and the set of $\operatorname{arcs} A=\{(i, j) \mid i, j \in V, i \neq j\}$. A nonnegative cost $c_{i j}$ associated with each $\operatorname{arc}(i, j) \in A$. The set of vertices (nodes) is partitioned into $k+1$ mutually exclusive nonempty subsets, called clusters, $V_{0}, V_{1}, \ldots, V_{k}$ (i.e. $V=V_{0} \cup V_{1} \cup \ldots \cup V_{k}$ and $V_{l} \cap V_{p}=\emptyset$ for all $l, p \in\{0,1, \ldots, k\}$ and $l \neq p$ ). The cluster $V_{0}$ has only one vertex 0 , which represents the depot, and remaining $\mathrm{n}$ nodes belonging to the remaining $k$ clusters represent geographically dispersed customers. Each customer has a certain amount of demand and the total demand of each cluster can be satisfied via any of its nodes. There exist $m$ identical vehicles, each with a capacity $Q$.

The generalized vehicle routing problem (GVRP) consists in finding the minimum total cost tours of starting and ending at the depot, such that each cluster should be visited by exactly once, the entering and leaving nodes of each cluster is the same and the sum of all the demands of any tour (route) does not exceed the capacity of the vehicle $Q$. An illustrative scheme of the GVRP and a feasible tour is shown in the next figure. 


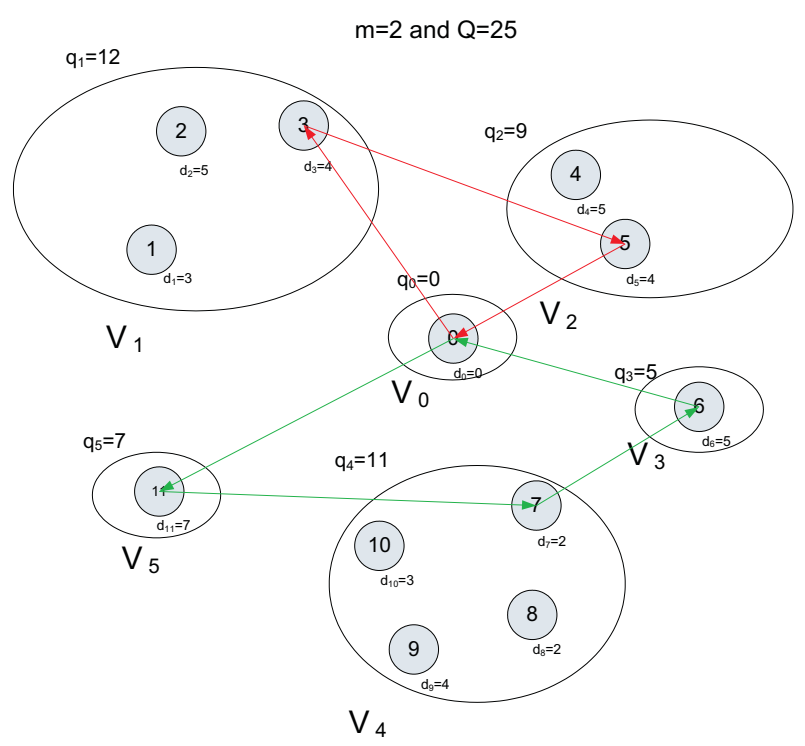

Figure 1 An example of a feasible solution of the GVRP

In Figure 1, it is presented a feasible solution consisting of a collection of two tours (routes): 0-3-5-0 and 0-11-7-6-0 satisfying the capacity restrictions and the condition that from each cluster is visited exactly one node. The cost of this feasible solution is obtained summing the costs of the arcs belonging to the selected tours.

The GVRP reduces to the classical Vehicle Routing Problem (VRP) when all the clusters are singletons and to the Generalized Traveling Salesman Problem (GTSP) when $m=1$ and $Q=\infty$. The GVRP is $N P$-hard because it includes the generalized traveling salesman problem as a special case when $m=1$ and $Q=\infty$.

Several real-world situations can be modeled as a GVRP. The post-box collection problem described in Laporte et al. [8] becomes an asymmetric GVRP if more than one vehicle is required. Furthermore, the GVRP is able to model the distribution of goods by sea to a number of customers situated in an archipelago as in Philippines, New Zeeland, Indonesia, Italy, Greece and Croatia. In this application, a number of potential harbours is selected for every island and a fleet of ships is required to visit exactly one harbour for every island. Several applications of the GTSP may be extended naturally to GVRP.

\section{Heuristic Algorithms for Solving the GVRP}

Two fundamental goals in computer science are finding algorithms with provably good run times and with provably good or optimal solution quality. A heuristic is an algorithm that abandons one or both of these goals; for example, it usually finds pretty good solutions, but there is no proof the solutions could not get arbitrarily bad; or it usually runs reasonably quickly, but there is no argument that this will always be the case. Heuristics are typically used when there is no known method to find an optimal solution, under the given constraints (of time, space etc.) or at all.

Several families of heuristic algorithms have been proposed for the classical VRP, see for example Laporte et al. [9]. These can be classified into two main classes: classical heuristics and metaheuristics. Most standard construction and improvement procedures in use belong to the first class. These methods performs a relatively limited exploration of the solution space and generally produce good quality solutions in reasonable computational times.

In what it follows we will provide three classes of heuristic algorithms for solving the GVRP: 
- constructive heuristics: Nearest Neighbour and a Clarke-Wright based heuristic;

- improvement heuristics: String Cross (SC), String Exchange (SE), String Relocation (SR) and String Mix (SM);

- a local-global heuristic.

\subsection{Constructive heuristics}

\section{Nearest Neighbour}

Perhaps the most natural heuristic for the GVRP is the famous Nearest Neighbour algorithm $(\mathrm{NN})$. In this algorithm the rule is always to go next to the nearest as-yet-unvisited customer subject to the following restrictions: we start from the depot, from each cluster is visited exactly one vertex (customer) and the sum of all the demands of the current tour (route) does not exceed the capacity of the vehicle $Q$. If the sum of all the demands of a current tour (route) exceeds the capacity of the vehicle then we start again from the depot and visit next the nearest customer from an unvisited yet cluster. If all the clusters are visited, then the algorithm terminates. A collection of routes traversing exactly one city from each cluster in the constructed order represents the output of the algorithm.

The nearest neighbour algorithm is easy to implement and executes quickly, but it can sometimes miss shorter routes, due to its greedy nature. The running time of the described nearest neighbour algorithm is $O\left(n^{2}\right)$.

\section{A Clarke-Wright based heuristic algorithm}

The Clarke and Wright [2] savings algorithm is perhaps the most well known heuristic for the VRP. It applies for the problems for which the number of vehicles is a decision variable, and works in the case of directed and undirected problems.

The algorithm in the case of the GVRP works as follows:

Step 1 (Savings computation). For each $i \in V_{l}$ and $j \in V_{p}$, where $l \neq p$ and $l, p \in\{1, \ldots, k\}$ compute the savings:

$$
s_{i j}=c_{i 0}+c_{0 j}-c_{i j} .
$$

It is obviously that $s_{i j} \geq 0$ and $s_{i j}=s_{j i}$. We order the savings in a nonincresing fashion.

At the beginning we create $k$ routes denoted $\left(0, i_{l}, 0\right), l \in 1, \ldots, k$ as follows for each cluster $V_{l}$ we define $c_{0 i_{l}}=\min \left\{c_{0 j} \mid j \in V_{l}\right\}$.

There will be as many routes as the number of clusters and total distance of the routes is:

$$
d=c_{0 i_{1}}+c_{0 i_{2}}+\ldots+c_{0 i_{k}} .
$$

Step 2 (Route extension). Consider in turn each route $(0, i, \ldots, j, 0)$. Determine the first saving $s_{u i}$ or $s_{j v}$ that can feasibly be used to merge the current route with another route ending with $(u, 0)$ or starting with $(0, v)$, for any $u \in V_{l}$ and $v \in V_{p}$, where $l \neq p$ and $l, p \in\{1, \ldots, k\}$ and $V_{l}$ and $V_{p}$ are clusters not visited by the route $(0, i, \ldots, j, 0)$.

Because at a given moment there can exist more feasible route extensions, the priority will have that one that produces the biggest reduction of the total distance of the route.

We implement the merge and repeat this operation to the current route. If no feasible merge exists, consider the next route and reapply the same operations.

Stop when no route merge is feasible.

The Clarke-Wright based algorithm for solving the GVRP is easy to implement and its running time is $O\left(n^{2} \log n\right)$. 


\subsection{Improvement heuristics}

The improvement heuristics algorithms for the GVRP are based on simple routes modifications and may operate on each vehicle route taken separately, or on a several routes at a time. In the first case, any improvement heuristic for Traveling Salesman Problem (TSP) can be applied, such as 2-OPt, 3-Opt, etc. In the second case, procedures that exploit the multi-route structure of the GVRP can be developed. We can see these improvements as a neighbourhood search process, where each route has an associated neighborhood of adjacent routes.

The heuristics algorithms for the GVRP that we are going to describe are based on the classification of the Van Breedam [16] of the improvement operations as string cross, string exchange, string relocation and string mix.

a) String cross $(S C)$ : two strings of vertices are exchanged by crossing two edges of two different routes.
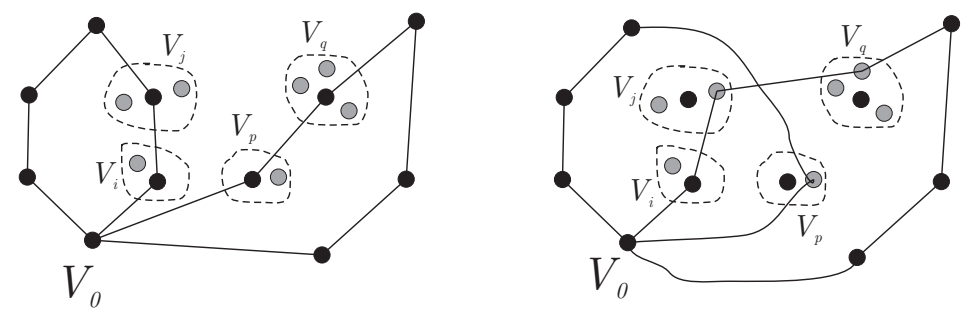

Figure 2 An example of a possible string cross. In the left side are presented the routes before the exchange of the vertices strings and in the right side the routes after the exchange

In the above picture there were presented just the clusters of the string of vertices that are exchanged in order to have a clearer figure. It is important to mention that we investigate all the possible connections of the exchanged vertices within the clusters in order to get improved routes, as is shown in Figure 2: the nodes belonging to the marked clusters after the exchange may be diferrent.

b) String exchange (SE): two strings of at most $r$ vertices are exchanged between two routes.
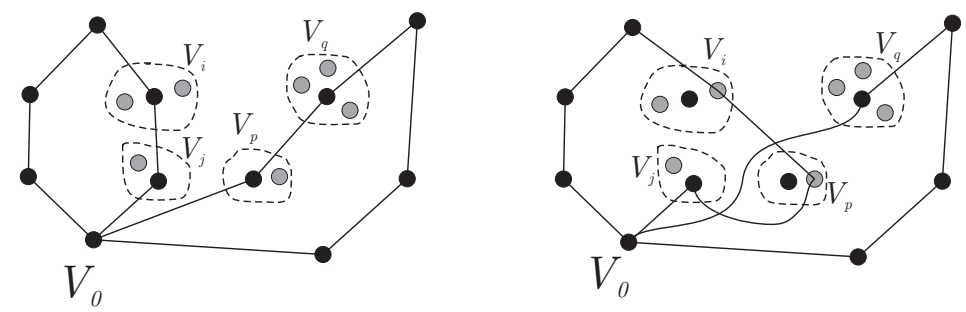

Figure 3 An example of a possible string exchange

c) String relocation $(S R)$ : a string of at most $k$ vertices is moved from one route to another $(k=1$ or $k=2)$.
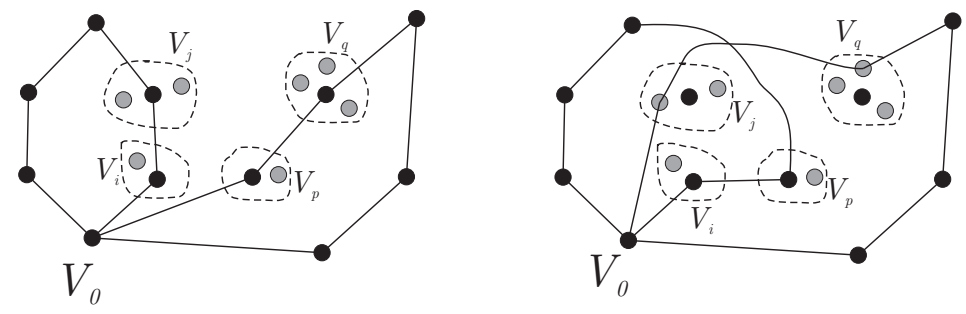

Figure 4 An example of a possible string relocation 
d) String mix (SM): consists in selecting the best move between the string exchange and string relocation.

\subsection{A local-global heuristic for GVRP}

The last heuristic algorithm for solving the GVRP that we are going to describe is based on local-global approach and it aims at distinguishing between global connections (connections between clusters) and local connections (connections between nodes from different clusters). As we will see, having a global collection of routes connecting the clusters it is rather easy to find the corresponding best (w.r.t. cost minimization) solution of the GVRP.

There are several generalized collection of routes, i.e. routes containing exactly one node from a cluster, corresponding to a global collection of routes. Between these generalized collection of routes there exist one called the best generalized collection of routes (w.r.t. cost minimization) that can be determined either by dynamic programming or by solving an linear integer program.

The local-global approach was applied succesfully to other generalized combinatorial optimization problems such as: generalized minimum spanning tree problem (GMSTP) and generalized traveling salesman problem (GTSP) in order to provide exact exponential time algorithms, strong mixed-integer programming formulations, solution procedures based on these mixedinteger programming formulations and a heuristic algorithm for solving the GMSTP, see [?,12].

Let $G^{\prime}$ be the graph obtained from $G$ after replacing all nodes of a cluster $V_{i}$ with a supernode representing $V_{i}$. We will call the graph $G^{\prime}$ the global graph. For convenience, we identify $V_{i}$ with the supernode representing it. Edges of the graph $G^{\prime}$ are defined between each pair of the graph vertices $V_{1}, \ldots, V_{k}$.

In the next figure we present the collection of generalized routes corresponding to the a global collection of routes.
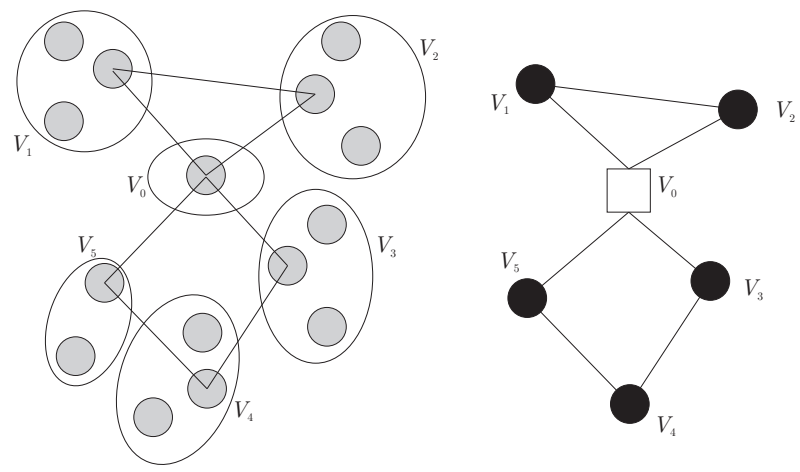

Figure 5 Example showing a generalized collection of routes corresponding to a global collection of routes

Given a global route and a sequence $\left(V_{0}, V_{k_{1}}, \ldots, V_{k_{p}}\right)$ in which the clusters are visited, we want to find the best feasible route $R^{*}$ (w.r.t cost minimization), visiting the clusters according to the given sequence. This can be done in polynomial time, by solving the following shortest path problem as we will describe below.

We construct a layered network, denoted by LN, having $p+2$ layers corresponding to the clusters $V_{0}, V_{k_{1}}, \ldots, V_{k_{p}}$ and in addition we duplicate the cluster $V_{0}$, containing the vertex denoted 0 and representing the depot. The layered network contains in addition the extra node denoted by $0^{\prime}$ for each. There is an arc $(i, j)$ for each $i \in V_{k_{l}}$ and $j \in V_{k_{l+1}}(l=1, \ldots, p-1)$, having the 
cost $c_{i j}$ and an arc $(i, h), i, h \in V_{k_{l}},(l=2, \ldots, p)$ having cost $c_{i h}$. Moreover, there is an arc $\left(i, 0^{\prime}\right)$ for each $i \in V_{k_{p}}$ having cost $c_{i 0^{\prime}}$.

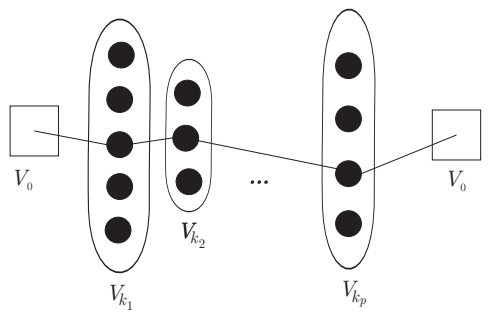

Figure 6 Example showing a route in the constructed layered network LN

We consider paths from 0 to $0^{\prime}$, that visits exactly one node from each cluster $V_{k_{1}}, V_{k_{2}}, \ldots, V_{k_{p}}$, hence it gives a feasible route.

Conversely, every route visiting the clusters according to the sequence $\left(V_{0}, V_{k_{1}}, \ldots, V_{k_{p}}\right)$ corresponds to a path in the layered network from 0 to $0^{\prime}$.

Therefore, it follows that the best (w.r.t cost minimization) route $R^{*}$ visiting the clusters in a given sequence can be found by determining all the shortest paths from 0 to the corresponding $0^{\prime}$ with the property that visits exactly one node from each of the clusters $\left(V_{k_{1}}, V_{k_{2}}, \ldots, V_{k_{p}}\right)$.

The overall time complexity of the above procedure is $O(m+\log n)$, where by $m$ we denoted the number of edges and $n$ number of nodes.

Therefore, given a global collection of routes connecting the clusters we can find efficiently the best corresponding collection of generalized routes. In order to provide global collections of routes we may use any improvement heuristics for the classical VRP.

\section{Conclusion and future work}

The Generalized Vehicle Routing Problem is an extension of the Vehicle Routing Problem (VRP) and consists in designing optimal delivery or collection routes, subject to capacity restrictions, from a given depot to a number of predefined, mutually exclusive and exhaustive node-sets (clusters). The GVRP is an NP-hard problem and finds many interesting real-world applications.

The aim of this paper was to present three classes of heuristic algorithms: constructive heuristics including Nearest Neighbour and a Clarke-Wright based heuristic; improvement heuristics including String Cross (SC), String Exchange (SE), String Relocation (SR) and String Mix (SM) and a local-global heuristic for the GVRP.

Computational results are planned in order to assess the effectiveness of the proposed heuristic algorithms.

Acknowledgement. This work was partially supported by CNCSIS-UEFISCSU, project number PNII - IDEI 508/2007.

\section{Bibliography}

[1] R. Baldacci, E. Bartolini and G. Laporte, Some applications of the Generalized Vehicle Routing Problem, Le Cahiers du GERAD, G-2008-82, 2008.

[2] G. Clarke and J.W. Wright, Scheduling of Vehicles From a Central Depot to a Number of Delivery Points, Operations Research, Vol. 12, pp. 568-581, 1964. 
[3] M. Fischetti, J.J. Salazar and P. Toth, A branch-and-cut algorithm for the symmetric generalized traveling salesman problem, Operations Research, 45:378-394, 1997.

[4] G. Ghiani, G. Improta, An efficient transformation of the generalized vehicle routing problem, European Journal of Operational Research, Vol. 122, pp. 11-17, 2000.

[5] I. Kara and T. Bektas, Integer linear programming formulation of the generalized vehicle routing problem, in Proc. of the 5-th EURO/INFORMS Joint International Meeting, 2003.

[6] I. Kara and P.C. Pop, New Mathematical Models of the Generalized Vehicle Routing Problem and Extensions, in Proc. of the International Conference on Applied Mathematical Programming and Modelling, Bratislava, Slovakia, May 27-30, 2008.

[7] G. Laporte, Location-routing problems, in B.L. Golden and A.A. Assad (eds.), Vehicle Routing: Methods and Studies, North-Holland, Amsterdam, pp. 163-197, 1988.

[8] G. Laporte, S. Chapleau, P.E. Landry and H. Mercure, An algorithm for the design of mail box collection routes in urban areas, Transportation Research B, Vol. 23, pp. 271-280, 1989.

[9] G. Laporte, M. Gendreau, J-Y. Potvin and F. Semet, Classical and modern heuristics for the vehicle routing problem, International Transactions in Operational Research, Volume 7, Issue 4-5, pp. 285 - 300, 2006.

[10] G. Nagy and S. Salhi, Location-routing issues: Models and methods, European Journal of Operational Research, Vol. 177, pp. 649-672, 2007.

[11] C.M. Pintea, D. Dumitrescu and P.C. Pop, Combining heuristics and modifying local information to guide ant-based search, Carpathian Journal of Mathematics, Vol. 24, No. 1, pp. 94-103, 2008.

[12] P.C. Pop, C. Pop Sitar, I. Zelina and I. Tascu, Exact algorithms for generalized combinatorial optimization problems, Lecture Notes in Computer Science, Vol. 4616, pp. 154-162, 2007.

[13] P.C. Pop, C.M. Pintea, I. Zelina and D. Dumitrescu, Solving the Generalized Vehicle Routing Problem with an ACS-based Algorithm, American Institute of Physics (AIP), Conference Proceedings: BICS 2008, Vol.1117, No.1, 157-162, 2009.

[14] P.C. Pop, Efficient Transformations of the Generalized Combinatorial Optimization Problems into Classical Variants, Proceedings of the 9-th Balkan Conference on Operational Research, Constanta, Romania, 2-6 September 2009.

[15] P.C. Pop, A survey of different integer programming formulations of the generalized minimum spanning tree problem, Carpathian Journal of Mathematics, Vol. 25, No. 1, pp. 104-118, 2009 .

[16] A. van Breedam, An analysis of the behavior of the heuristics of the vehicle routing problem for a selection of problems, with vehicle-related, customer-related and time-related constraints, Ph.D. Dissertation, University of Antwerp, Belgium, 1994. 